\title{
Evaluating post-operative venous thromboembolism risk in urology patients using a validated risk assessment model
}

\author{
Winston Paul René Padayachee ${ }^{\text {*}^{*}}$, Mohamed Haffejee ${ }^{1}$ and Marietha $\mathrm{Nel}^{2}$
}

\begin{abstract}
Background: Venous thromboembolism (VTE) is an important cause of post-surgical morbidity and mortality. This study aimed to apply a validated risk assessment model to evaluate the risk of post-operative VTE in urology patients.

Methods: This prospective descriptive observational study used the Caprini risk assessment model to evaluate VTE risk in patients planned for elective urology surgery at a tertiary Johannesburg hospital from January to June 2020.

Results: Two hundred and twenty-six patients with a mean age of 52 years were evaluated for post-operative VTE risk. The population was generally overweight, with a mean BMl of $26.3 \mathrm{~kg} / \mathrm{m}^{2}$. The mean Caprini score was 4.42 , reflecting a population at high risk for post-operative VTE. There was no statistically significant difference between males and females in this regard. On average, participants had three risk factors for post-operative VTE. Fifteen per cent of all patients were at low risk for VTE, while $40.3 \%$ of participants were categorised as moderate risk. The category with the highest percentage of participants (44.7\%) was the high-risk category (Caprini score $\geq 5$ ). High-risk patients undergoing oncology surgery comprised $16.8 \%$ of the population, and these patients may require extended duration pharmacological thromboprophylaxis to prevent VTE. The most clinically significant risk factors for post-operative VTE included age, obesity, malignancy and HIV infection.

Conclusion: Venous thromboembolism may be difficult to diagnose, and clinicians may underestimate the risk for it to develop. Risk assessment models, such as the Caprini score, are objective and a practical tool to guide the application of thromboprophylaxis. The application of the Caprini RAM in the elective urological surgery population at Chris Hani Baragwanath Academic Hospital yields similar results to studies performed elsewhere on similar surgical populations. Further research is required to evaluate whether the actual incidence of VTE correlates with the risk assessment in this population. Clinician compliance with the use of RAMs as well as the corresponding recommendations for prophylaxis may need to be evaluated. A validated risk assessment model which accounts for procedure-specific risks in urology may be useful.
\end{abstract}

Keywords: Venous thromboembolism, Urology, Caprini, Risk assessment model

*Correspondence: winstonpadayachee@gmail.com

${ }^{1}$ Division of Urology, Department of Surgery, School of Clinical Medicine, Faculty of Health Sciences, University of the Witwatersrand, Johannesburg, South Africa

Full list of author information is available at the end of the article

\section{Background}

In 1856, Rudolph Virchow established the foundations upon which modern understanding of venous thromboembolism is based. Although his description of hypercoagulability, stasis and endothelial damage appears to originate from his work on the consequences rather than the origins of pulmonary embolism, it has become 
popularised as 'Virchow's Triad'-the pathological basis for the development of thrombosis and emboli [1].

Venous thromboembolism (VTE) is a collective term encompassing deep vein thrombosis and pulmonary embolism. Although it may occur elsewhere, deep vein thrombosis (DVT) usually refers to the development of a thrombus in the deep veins of the lower limbs. The thrombus commonly originates in the calf (calf DVT) and may extend proximally (proximal DVT). Symptoms of DVT typically occur once the proximal veins are involved. Thrombi may embolise to cause a pulmonary embolism (PE). Both DVT and PE may be asymptomatic or symptomatic and may occur as a first episode or as a recurrence $[2,3]$.
In 2005, Dr Joseph A Caprini published a risk assessment model (RAM) to evaluate an individual's risk for post-operative VTE (Fig. 1 and Table 1). The 9th edition of the American College of Chest Physicians' (ACCP) "CHEST" guidelines on non-orthopaedic VTE (2012)

Table 1 Risk of post-operative VTE according to Caprini score [5]

\begin{tabular}{ll}
\hline Caprini score & Risk level \\
\hline $0-1$ & Low risk \\
2 & Moderate risk \\
$3-4$ & High \\
5 or more & Highest \\
\hline
\end{tabular}

\section{Thrombosis Risk Factor Assessment}

Patient's Name:

\section{Choose All That Apply}

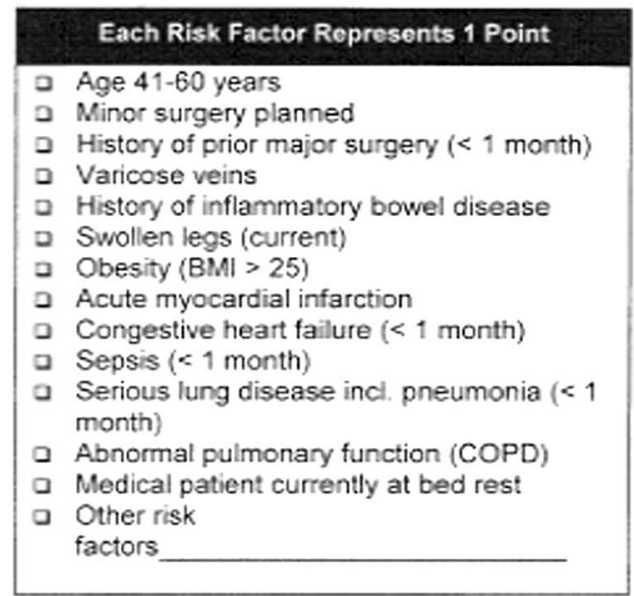

\section{Each Risk Factor Represents 3 Points}

D Age over 75 years
D History of DVT/PE
a Family history of thrombosis*
a Positive Factor $V$ Leiden
a Positive Prothrombin $20210 \mathrm{~A}$
a Elevated serum homocysteine
a Positive lupus anticoagulant
a Elevated anticardiolipin antibodies
a Heparin-induced thrombocytopenia (HIT)
a Other congenital or acquired thrombophilia
If yes:
Type
-most frequently missed risk factor

Fig. 12005 Caprini risk assessment model [5]
EVANSTON NORTHWESTERN HEALTHCARE.

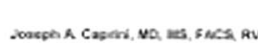

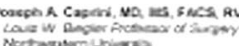

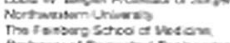

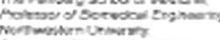

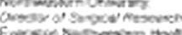

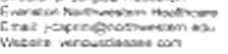

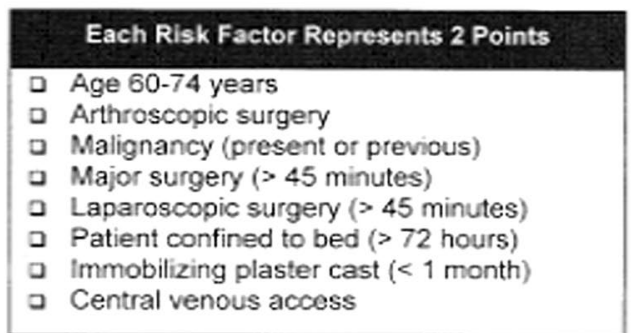

\section{Each Risk Factor Represents 5 Points}

- Elective major lower extremity arthroplasty

a Hip, pelvis or leg fracture (<1 month)

a Stroke (< 1 month)

a Multiple trauma (< 1 month)

a Acute spinal cord injury (paralysis) $(<1$ month)

\section{For Women Only (Each Represents 1 Point)}

- Oral contraceptives or hormone replacement therapy

- Pregnancy or postpartum ( $<1$ month)

- History of unexplained stillborn infant, recurrent spontaneous abortion $(\geq 3$ ). premature birth with toxemia or growthrestricted infant

Total Risk Factor Score 
recommends the use of the 2005 Caprini RAM for VTE risk assessment [4].

Since VTE is an important preventable cause of postsurgical morbidity and mortality, this study aimed to investigate the most prevalent risk factors for VTE, as well as the overall VTE risk in South African patients undergoing elective urological surgery.

\section{Methods}

The purpose of this study was to evaluate the risk of postoperative VTE in patients undergoing surgery for urological disease in an African hospital environment and to determine the most prevalent risk factors for VTE in this population. This prospective, descriptive observational study was conducted at Chris Hani Baragwanath Academic Hospital (CHBAH) in Soweto, Gauteng Province, South Africa.

The study population included adult patients planned for elective urology surgery from January-June 2020. Patients planned for day surgery under local anaesthetic were excluded, as were patients already on therapeutic anticoagulation. Convenience sampling was applied to the above population to evaluate the baseline risk for venous thromboembolism. The patients were all evaluated pre-operatively using the 2005 Caprini RAM. The risk of bias was low, given that patient admission dates are independent of surgical procedure or demographics, and therefore independent of risk factors for VTE.

Clinical endpoints evaluated included the following: patient age and gender, proposed surgery, risk factors for VTE (as per Caprini 2005 RAM, Fig. 1) and total Caprini score and risk level (Table 1).

\subsection{Ethics approval and consent to participate}

Information was collected by interviewing/examining patients and reviewing available clinical records. Written informed consent from study participants was obtained. This study was approved by the University of Witwatersrand Human Research Ethics Committee (protocol no: M191175). Permission for the study was obtained from the relevant clinical head of department and the CHBAH chief executive officer. No identifying personal particulars of participants were collected, and all records are kept secure by the investigator.

\section{Data analysis}

Data were recorded in an Excel datasheet and imported into STATA version 14 (Stata Corp, 2018) statistical software and coded into numbers for subsequent analysis. The analysis was done using a stepwise approachdescriptive statistics in the form of frequency tables and graphs, followed by Chi-square tests on independence between two variables and Spearman's correlation to test for associations between risk factors and demographics. Analysis was done using STATA software and at a 95\% confidence interval with a $5 \%$ significance.

\section{Results}

\subsection{Demographic and anthropometric findings}

The study results describe the risk profile for VTE in 226 adult patients (163 male; 63 female) planned for elective surgical admission to the urology unit at Chris Hani Baragwanath Academic Hospital from January 2020 to June 2020. The youngest participant was 18 years of age, while the oldest was 86 years. The mean age of participants was 52.12, with a normal distribution above and below the mean $(\mathrm{SD}=16.3)$.

The mean BMI of the participants was $26.3 \mathrm{~kg} / \mathrm{m}^{2}$ ( $\mathrm{SD}=6.7$ ), which ranged from 21.8 (IQR: $25 \%$ ) to 30.1 (IQR:75\%). Patients in this sample are generally overweight [6]. Proportionally, male participants were slightly more likely to have a healthy BMI of $19-24.9 \mathrm{~kg} / \mathrm{m}^{2}$ (39.3\%) compared to females (30.2\%).

\subsection{Planned surgery}

As per the Caprini RAM, major surgery was defined as a procedure estimated to last longer than $45 \mathrm{~min}$, while minor surgery would last less than $45 \mathrm{~min}$. Overall, the precise nature of surgery in the sample population varied widely. As a result, the prevalence of many operations was very low and not further statistically analysed. Of the 226 urology patients planned for elective surgery in this sample, $83 \%$ were not planned to undergo surgery for non-cancerous conditions, with $17 \%$ planned for oncology surgery. $31 \%$ underwent open major surgery, $9 \%$ had open minor surgery, $38 \%$ had endoscopic major surgery, and $22 \%$ endoscopic minor surgery.

\subsection{Total Caprini Score}

Figure 1 demonstrates the Caprini RAM. Risk factors are weighted according to their odds ratios for contributing to VTE-falling in categories which contribute either 1, 2, 3 or 5 points to the patients score. Based on the frequency and weighting of risk factors, Fig. 2 reveals the mean contribution for each category towards the total Caprini score in this population. The mean Caprini score was 4.42, reflecting a population at high risk for post-operative VTE. The mean score was slightly higher for female $(4.47, \mathrm{SD}=1.79)$ compared to male (4.39, $\mathrm{SD}=1.8)$ patients. This difference is not statistically significant at $5 \%$ level.

The mean total Caprini score was 6.71 for patients planned to undergo oncology surgery, and lower $($ mean $=3.93)$ for patients undergoing non-oncology surgery. This difference is slightly more than the expected additional two points that every oncology patient garners 


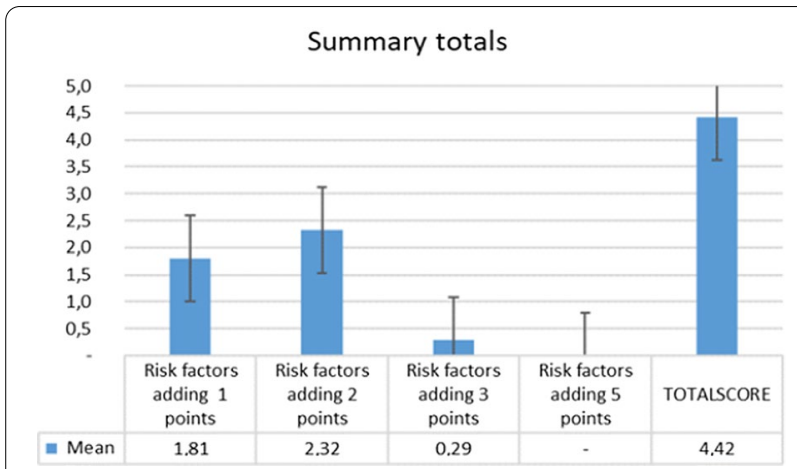

Fig. 2 Mean contribution from each risk factor category and total Caprini score in the sample population

for malignancy itself as a risk factor, according to the RAM.

\subsection{Number of risk factors per person}

All patients had at least one risk factor-planned surgery. If surgery is excluded, $8.8 \%$ of patients had no additional risk factors, while $21.6 \%$ had only one additional risk factor. The most frequent number of additional risk factors per person was two (35.4\%), while a third of participants (33.6\%) had three or more additional risk factors. The mean number of additional risk factors per participant was 3.06 , when excluding surgery as a risk factor.

\subsection{Calculated VTE risk}

The Caprini RAM infers whether a patient is at low, moderate or high risk for VTE based on the patients total Caprini score (Table 1). Additionally, the ACCP guideline for VTE prophylaxis recommends extended duration prophylaxis for high-risk patients undergoing oncology surgery, and these patients are therefore categorised separately in this study. Figure 3 demonstrates the distribution of risk for VTE in the study population.

\subsection{Sub-stratification of high-risk patients}

In this study, nearly a third (29.2\%) of all participants had a high-risk score of 5-6. More than a tenth of the population $(13.7 \%)$ had a high-risk score of $7-8$, while only $1.8 \%$ had a score of more than 8 .

\subsection{Most clinically significant risk factors}

Figure 4 demonstrates the ten risk factors in the population, which most significantly contribute to the total Caprini score. Since risk factors are weighted differently, clinical significance is inferred by the frequency with which these risk factors occurred, multiplied by their weighting in the risk assessment model. From this list, the potentially modifiable risk factors are obesity, HIV

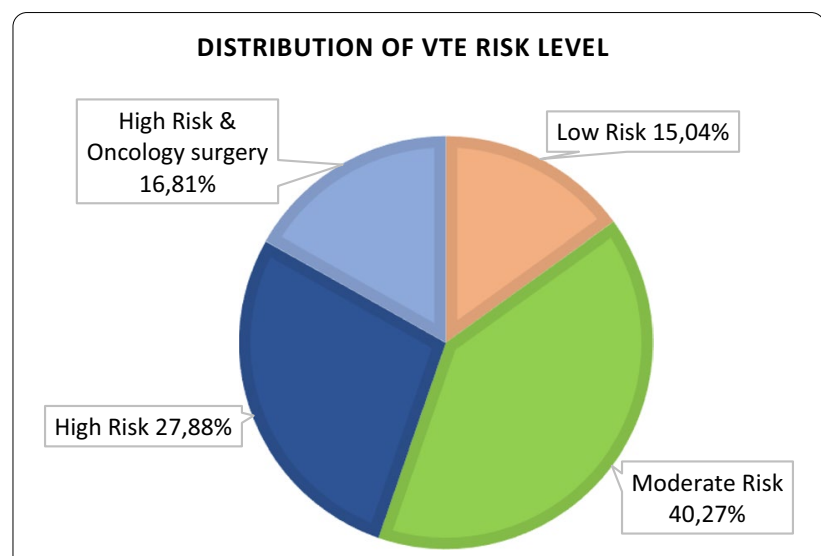

Fig. 3 Risk level for VTE within the sample population

infection, varicose veins and swollen legs. Malignancy is not considered to be a modifiable risk factor since many of the patients with this risk factor were presenting for surgery for that same malignancy.

\section{Discussion}

Venous thromboembolic disease is often asymptomatic, may be difficult to diagnose, and in the case of pulmonary embolism, may present as sudden death. Hence, determining the incidence of VTE in the general population can be challenging, and it is usually underestimated [7]. Despite this, VTE remains, statistically, the third most common cardiovascular disease after myocardial infarction and stroke $[3,8]$.

The VTE impact assessment group in Europe (VITAE) found that $56 \%$ of DVT and $70 \%$ of PE were hospital acquired. Seventy-one per cent of VTE-related deaths were hospital acquired. However, only $7 \%$ of all deaths occurred in patients already diagnosed with disease [9], which is an affirmation of the significance of VTE as a preventable cause of hospital deaths, and the importance of risk assessment and prophylaxis. The 1-month mortality is around $6 \%$ for DVT and $12 \%$ for PE [10]. The 3-month all-cause mortality from PE ranges between 8.3 and $17 \%$ [8]. PE is the most common cause of preventable death in hospital inpatients (10\%) [11]. There are approximately 150,000-200,000 VTE-related annual deaths in the USA. Of these, about 1/3 involves disease acquired post-surgery [4]. In Canada, about 20\% of VTE is associated with surgery within 3 months [12]. Around $64 \%$ of hospitalised surgical patients are at risk [8].

There is a paucity of literature evaluating the risks of VTE in African and South African hospital populations, and there are no large-scale studies based on the general population. A 2017 systematic review estimated the risk of DVT post-surgery in Africa as between 2.4 and 9.6\%, 
Ten most significant contributors to total Caprini score (frequency $x$ weighting)

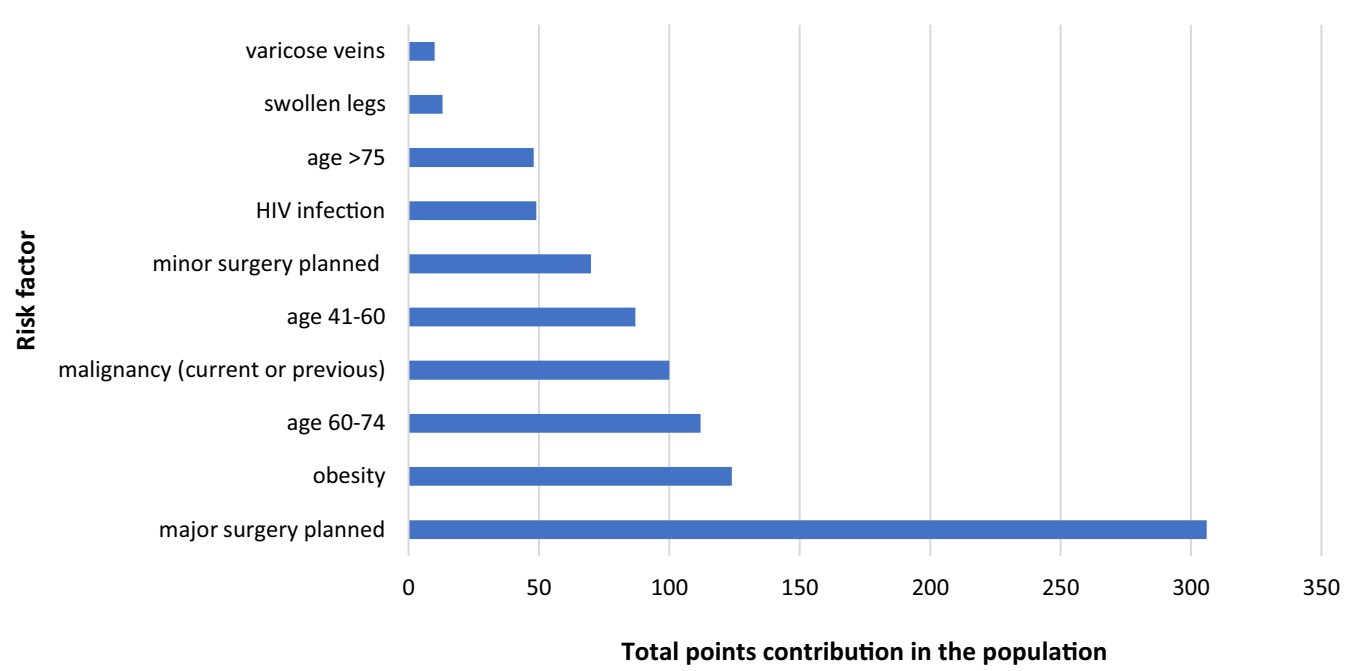

Fig. 4 Most clinically significant risk factors in the sample population considering frequency and weighting

with the lowest risk identified in a sub-Saharan population. Limited data exists on the risk of PE following surgery in Africa [13].

There are significant healthcare costs derived from the diagnosis and management of a primary VTE, but also from complications such as post-thrombotic syndrome (approximately 33-50\%), pulmonary hypertension $(4-5 \%)$ and VTE recurrence $[8,9]$. More than half of patients have chronic complications 4 years after a pulmonary embolism. [8]. Based on the incidence, the difficulty in diagnosis, and the impact with regard to quality of life and healthcare costs, multiple public health institutes have identified VTE disease as an important public health concern. VTE requires increased patient and clinician awareness, research on prevention, and implementation of evidence-based policies for risk assessment and prevention [7].

\subsection{Validation of the Caprini RAM}

The risk assessment model has been validated for use in general, vascular and urologic surgery [14], plastic and reconstructive surgery [15], otolaryngology surgery [16], surgical critical care [17] and thyroid and parathyroid surgery [18]. However, it is still criticised for failing to discriminate well between different types of surgery. Newer versions of the Caprini RAM exist, but these have not yet been as extensively validated.

Some validation studies have included post-urological surgery patients within mixed surgical populations. In one study specifically evaluating individual's post-robotassisted radical prostatectomy, VTE was found in $1.4 \%$ of the population, and the use of the Caprini RAM was supported. Specifically, a score of 6 or more was predictive of VTE [19]. However, another study reporting on procedure-specific VTE risk has criticised the Caprini RAM for failing to adequately discriminate the risk between different types of urological surgery [20].

In 2017, Pannuci et al. published a meta-analysis of 13 studies investigating the benefits and risks associated with pharmacological thromboprophylaxis based on a 2005 Caprini risk stratification. The study found that the risk of VTE in patients with a score of 3-4 was $0.7 \%$. This increased to $1.8 \%$ for scores of $5-6$ and $4 \%$ for scores of $7-8$. A Caprini score of $>8$ was associated with a $10.7 \%$ incidence of VTE. The risk reduction for VTE in patients with scores of $7-8$ or $>8$ was significant (odds ratios 0.6 and 0.4 , respectively). There was no significant reduction in risk among patients with scores of 6 or lower, and the risk of bleeding was increased in all patients [21]. This appears to support the use of the Caprini model for risk stratification, and supports the adoption of individualised approaches to pharmacological thromboprophylaxis.

\subsection{The risk of post-operative venous thromboembolism}

This study intended to determine the risk level for VTE in patients undergoing elective urology surgery at $\mathrm{CHBAH}$, using the Caprini RAM. The mean Caprini score of 4.42 describes a population that is generally at high risk for developing post-operative VTE (Table 2).

This study demonstrates that urology patients undergoing non-oncology surgery are on average, at moderate risk for post-operative VTE. On the other hand, urology 
Table 2 Probability (\%) of different risk categories for VTE in this population compared with other studied populations

\begin{tabular}{|c|c|c|c|c|c|}
\hline Publication & Surgical population & Very low risk (0) & Low risk (1-2) & $\begin{array}{l}\text { Moderate } \\
\text { risk (3-4) }\end{array}$ & High risk $(\geq 5$ \\
\hline \multirow[t]{3}{*}{ This study* } & Urology elective surgery & 0.0 & 15.04 & 40.27 & 44.69 \\
\hline & Urology oncology surgery & 0.0 & 0.0 & 2.56 & 97.44 \\
\hline & Urology non-oncology surgery & 0.0 & 18.18 & 48.13 & 33.69 \\
\hline McAlpine et al. [20] & Urology major abdominal or pelvic surgery & 0.01 & 0.82 & 8.87 & 90.29 \\
\hline Bahl et al. [14] & General, vascular and urology surgery & & $11.37^{\mathrm{a}}$ & 36.53 & 52.11 \\
\hline Barber et al. [22] & Gynaecology oncology surgery & 0.0 & 0.1 & 2.98 & 96.92 \\
\hline Snyman et al. [23]* & Elective Gynaecology surgery & & $17^{\mathrm{a}}$ & 38 & 45 \\
\hline Jacobson et al. (2014)* & Unspecified surgical inpatients & & $20.3^{\mathrm{a}}$ & 15 & 43.7 \\
\hline Macht et al. [18] & Thyroid and parathyroid surgery & & $72^{\mathrm{a}}$ & 26 & 2 \\
\hline Shuman et al. [16] & Otolaryngology surgery & & $11.4^{\mathrm{a}}$ & 31 & 57 \\
\hline Bilgi et al. [24] & Elective and emergency general surgery & & $28.2^{\mathrm{a}}$ & 29.5 & 42.2 \\
\hline
\end{tabular}

*South African data

${ }^{\text {a }}$ These studies made use of risk-level terminology that differs from the ACCP guideline. Hence, low and very low risk categories are combined to align the categories with the equivalents from other studies

patients undergoing oncology surgery are at high risk for post-operative VTE, due to active malignancy and because they are likely to be older.

\subsection{Recommendations for thromboprophylaxis in urology} The American Urological Association (AUA) found insufficient high-quality evidence to issue a formal guideline regarding thromboprophylaxis. Their 2008 best practice statement adopted a risk assessment model published by Geerts et al., which categorised patients into low, moderate, high or very high risk. This was based on age, major versus minor surgery, and the presence of additional risk factors. General recommendations were made for the use of mechanical and/or pharmacological thromboprophylaxis based on these risk levels. Recommendations for different categories of surgery were also made. Additionally, limited recommendations were made regarding extended duration prophylaxis [25].

The European Association of urology (EAU) published a guideline in March 2017, primarily based on the findings of the Risk of Thrombosis and Bleeding in Urological Surgery (ROTBUS) systematic review. They made use of an un-validated risk assessment model. A number of procedure-specific recommendations were made, but all are based on low or very low quality supporting evidence. Recommendations were based on initiation of prophylaxis the day after surgery, and weeks was the recommended duration for pharmacologic prophylaxis [26].

No other society has developed guidelines for thromboprophylaxis specific to urology. Other important international VTE guidelines, however, may be applicable to patients undergoing urological surgery. These include those developed by the American College of Chest
Physicians (ACCP), National Institute for Health and Care Excellence (NICE), National Comprehensive Cancer Network (NCCN), German Association of Scientific Medical Societies (AWMF) and the Australian National Health and Medical Research Council (AMH-MRC) [27].

For the purpose of this study, the ACCP guideline for non-orthopaedic surgical patients was used to determine the need for thromboprophylaxis, specifically the recommendations for patients undergoing general and abdomino-pelvic surgery. This publication explicitly includes urology patients in this category. The Caprini RAM is used, and recommendations are made based upon the results [4].

It should be noted that the terminology used to describe risk levels differs between the original 2005 Caprini RAM and the ACCP guideline. For the purposes of this study, the ACCP terminology was used.

Data from this study suggest that most patients undergoing elective urology surgery at $\mathrm{CHBAH}(85 \%)$ are at moderate or high risk for VTE and therefore may require pharmacological prophylaxis, depending on their risk of bleeding. The remaining patients should ideally be prescribed mechanical prophylaxis in the post-operative period. The total absence of 'very low risk' patients in this population is due to every patient scoring at least one point on the Caprini RAM for planned elective surgery.

Guidelines recommend that when feasible, early postoperative ambulation is standard of care for all patients for VTE prevention [4, 11]. Other mechanical and pharmacological methods are recommended based on patients' risk profiles. The ACCP recommendations (Table 3) for prophylaxis are based on the estimated risk of developing post-operative VTE in the absence of any 
Table 3 Risk levels for VTE and recommended prophylaxis as per ACCP [4]

\begin{tabular}{|c|c|c|c|}
\hline Risk level & Caprini score & Recommended thromboprophylaxis & $\begin{array}{l}\text { Need for prophylaxis in } \\
\text { elective urology patients at } \\
\text { CHBAH (\%) }\end{array}$ \\
\hline Very low & 0 & Early ambulation & 0 \\
\hline Low & $1-2$ & $\begin{array}{l}\text { Mechanical prophylaxis: intermittent pneumatic compression (IPC) pre- } \\
\text { ferred over elastic stockings (ES) }\end{array}$ & 15.04 \\
\hline Moderate & $3-4$ & $\begin{array}{l}\text { No high risk for major bleeding } \\
\text { Low molecular weight heparin (LMWH) or low-dose unfractionated heparin } \\
\text { (LDUH) or mechanical prophylaxis } \\
\text { High risk for major bleeding } \\
\text { Mechanical prophylaxis }\end{array}$ & 40.27 \\
\hline High & $\geq 5$ & $\begin{array}{l}\text { No high risk for major bleeding } \\
\text { LMWH or LDUH in addition to mechanical prophylaxis } \\
\text { High risk for major bleeding } \\
\text { Mechanical prophylaxis } \\
\text { LMWH or LDUH once risk of bleeding subsides }\end{array}$ & 27.88 \\
\hline $\begin{array}{l}\text { High with abdomino- } \\
\text { pelvic oncology surgery }\end{array}$ & $\geq 5$ & $\begin{array}{l}\text { No high risk for major bleeding: LMWH or LDUH in addition to mechanical } \\
\text { prophylaxis. Consider extended duration pharmacologic thromboprophylaxis } \\
\text { for } 4 \text { weeks }\end{array}$ & 16.81 \\
\hline
\end{tabular}

prophylaxis for general, urology and vascular surgery patients: low risk $1.5 \%$; moderate risk $3 \%$; and high risk $6 \%[4]$.

\subsection{Risk of bleeding}

Pharmacological thromboprophylaxis represents an increase in the risk of major post-operative bleeding of approximately $50 \%$ [4]. The treating clinician must therefore weigh up the risk of post-operative bleeding in comparison with the risk of post-operative VTE. This study assessed patients pre-operatively, and thus the risk of bleeding was not determined, as this is conventionally evaluated at the time of surgery.

The ACCP defines a "high risk for major bleeding" as a $2 \%$ risk of post-operative bleeding. This is specific to the nature of the surgery. Patients are also considered to have a high bleeding risk if the consequences of bleeding would be particularly severe-for example following spinal surgery. In addition, clinicians should consider patient factors such as thrombocytopaenia or any other coagulopathy, renal or liver failure, uncontrolled hypertension, prior heparin induced thrombocytopaenia, or a prior history of bleeding associated with surgery $[4,28]$.

\subsection{Venous thromboembolism and bleeding in urology surgery}

Sixty per cent of the sample population at $\mathrm{CHBAH}$ were planned to undergo endoscopic surgery, with the remaining patients undergoing open surgery. This may result in an overestimation of the risk of VTE according to the Caprini RAM as the endoscopic procedure may represent a lesser surgical insult. While some validation studies have included urological patients along with general surgical patients [14], and other studies have validated the RAM for a specific urological procedure [19], no study validating the use of the Caprini RAM in a heterogeneous, but exclusively urological population yet exists.

The 2005 Caprini RAM does not distinguish between endoscopic and open surgery-only between minor and major surgery according to duration. However, the majority (62.5\%) of urological endoscopic procedures at $\mathrm{CHBAH}$ are major procedures lasting longer than $45 \mathrm{~min}$. Therefore, these procedures still incur a significant risk for the development of VTE on account of venous stasis due to immobility, calf muscle paralysis, venous over-distension and surgical insult producing endothelial damage, as well as hypercoagulability due to surgical stress.

In addition, urological endoscopic procedures are almost always performed in the lithotomy position, which when compared with the supine position, reduces lower extremity venous blood flow by up to $40 \%$, even when pneumatic compression devices are used [29].

The @RISTOS study prospectively evaluated the incidence of VTE in patients undergoing urologic surgery for cancer. The incidence of post-operative bleeding was $17.1 \%$ in those who received pharmacological prophylaxis compared to $5.7 \%$ in those who did not. The incidence of symptomatic VTE was $0.87 \%$, which was lower than the gynaecological and general surgical populations (2-2.1\%) evaluated, primarily on account of the greater proportion of endoscopic procedures performed in urology. Other favourable factors in urology patients included more procedures performed under regional anaesthesia, procedures less than two hours in duration, and a lesser frequency of advanced cancer [30]. 
Clemént et al. assessed the incidence of asymptomatic and symptomatic VTE in cancer patients after radical/ partial nephrectomy, radical cystectomy and radical prostatectomy. Radical cystectomy was found to pose a significantly higher risk for early post-operative VTE when compared to the other procedures [31]. Alberts et al. found the incidence of VTE post-urology surgery for cancer to be $1.4 \%$ at 30 days. They confirmed that radical cystectomy represents the greatest risk for VTE and that minimally invasive surgery lowered the risk. The strongest factors associated with the development of VTE were BMI $>30 \mathrm{~kg} / \mathrm{m}^{2}$, prolonged surgical time, radical cystectomy and metastatic cancer [32].

The ROTBUS systematic review analysed the risk of VTE and bleeding following urologic surgery. For cancer surgery, radical cystectomy represented a risk for four weeks post-operative VTE of $2.6-11.6 \%$ regardless of the patient's baseline risk, but was associated with a low risk of bleeding $(0.3 \%)$. The risk for VTE after radical prostatectomy ranged from 0.2 to $15.7 \%$, with the highest incidence of VTE among those undergoing pelvic lymphadenectomy. Robotic and laparoscopic procedures were found to have a lower risk for thrombosis when compared to open prostatectomy. The overall risk of bleeding following prostatectomy was low. Kidney surgery for cancer was found to represent a risk for VTE in $0.7-11.6 \%$ of patients, with the greatest risk in those patients with a baseline high risk for VTE. For kidney surgery, the risk of bleeding was $0.1-2 \%$. The risk for VTE after radical penectomy with inguinal lymph node dissection was $0.8-3.1 \%[33,34]$.

Taking the above into account, the greatest benefit for thromboprophylaxis, when assessing the risk of bleeding versus the risk for VTE in open urological cancer surgery, appears to be in patients following radical cystectomy or open radical prostatectomy.

For non-cancer urological surgery, ROTBUS found that good quality evidence for procedure-specific risks of VTE and bleeding was lacking. The study suggested that the net benefit for thromboprophylaxis appeared greater for some procedures such as renal transplantation, but that the risk of bleeding may outweigh the risk of VTE for certain procedures such as transurethral resection of the prostate (TURP) [35]. VTE rates post-TURP are generally low $(0-0.4 \%)$, and the procedure is associated with post-operative bleeding [36].

TURP was a frequently planned surgery among the sample population at CHBAH (11.1\%), and these patients require careful consideration for thromboprophylaxis based on their Caprini score, and the clinicians perceived risk of post-operative bleeding.

The ROTBUS study also found that around 50\% of major post-operative bleeds occur between surgery and the following morning. The majority (90\%) of bleeds occur within four days. The risk of VTE, however, was consistently elevated during the first four weeks following surgery $[34,35]$. The net benefit of prophylaxis, therefore, may be greater a few days after surgery. This may aid a clinician's decision to delay post-operative prophylaxis in procedures with a high bleeding risk, and/or to continue prophylaxis for an extended duration.

Despite the relatively lower overall incidence of VTE in urology patients, the mortality for symptomatic VTE is around $58 \%$. The rates of clinical and subclinical VTE after radical cystectomy are among the highest of any type of surgery [30, 36]. Hence, post-operative VTE should be considered an important preventable cause of death following urologic surgery.

Among observational studies evaluating the risks of thrombosis and bleeding, the usage and duration of thromboprophylaxis varied widely. Reporting on thromboprophylaxis use was also inconsistent. Nevertheless, the analysis of baseline risk and procedure-specific risk may assist clinicians in decision-making. It is clear that the population undergoing urologic surgery is highly heterogeneous and further high-quality evidence is needed to evaluate procedure-specific risks.

\subsection{Sub-stratification of high-risk patients}

The original Caprini model categorised all patients with scores of 5 or more at the same risk level. Subsequent validation studies demonstrated that the risk of VTE continues to increase with cumulative score within this high-risk category. In particular, statistically significant differences were noted in these studies between patients with scores of 5-6, 7-8 and $>8$. Bahl et al. studied general, vascular and urological surgery patients, finding that the $70.4 \%$ of their high-risk patients had scores of $5-6$, while $23.5 \%$ had scores of $7-8$ and $6.1 \%$ had scores of 9 or more. When they further analysed the incidence of VTE in their population they found that the incidence of VTE roughly doubled from each of these categories to the next (1.3\% vs $2.6 \%$ vs 6.5\%) [14]. Consequently, these categories are recognised as separate entities in most current applications of the RAM. Different authors appear to use different terminology when naming these categories, i.e., low risk, moderate risk, high risk, highest risk, superhigh risk $[14,15,17]$. As such, describing these groups by the actual numerical Caprini scores may be preferred.

Among patients undergoing elective urology surgery at $\mathrm{CHBAH}, 45 \%$ of the population were considered high risk for post-operative VTE (Caprini score $\geq 5$ ). If one was to sub-stratify this high-risk population $(\mathrm{n}=101)$, $65 \%$ had scores of $5-6$, while $31 \%$ had scores of $7-8$ and only $4 \%$ scored 9 or more. Since more than a third of high-risk patients at $\mathrm{CHBAH}$ may have a greater than 
expected risk of VTE, it is clear that clinicians should not only consider the risk level of the patient, but also the total Caprini score. Although different sub-categories of high-risk patients have thromboprophylaxis applied in the same manner according to guidelines, the accelerated risk of VTE with an increase in Caprini score may alter a clinician's decision, when weighing up the benefit of anticoagulation with the risk of bleeding. In addition, high-risk patients with higher total Caprini scores may be considered for extended duration thromboprophylaxis.

\subsection{Extended duration thromboprophylaxis}

Extended duration thromboprophylaxis is not routinely practiced at the urology unit at CHBAH. Seventeen per cent of urology patients at $\mathrm{CHBAH}$ were scored as high risk for VTE while also undergoing surgery for cancer. The evidence suggests that these patients may benefit from extended duration pharmacological thromboprophylaxis. More than $50 \%$ of VTE post-surgery occurs after discharge [35, 37]. Hence, the appropriate duration for post-operative pharmacological thromboprophylaxis is questioned.

A South African guideline recommends that in general, where indicated, low molecular weight heparin (LMWH) should be continued post-surgery until the patient is fully mobile. In high-risk patients a total of seven to ten days of prophylaxis is recommended. For patients undergoing major cancer surgery, five weeks of therapy is recommended, whereas hip and knee surgery has recommendations of five and two weeks, respectively [11].

The ACCP reviewed four studies on extended duration prophylaxis, finding that its use in high-risk patients reduced the incidence of non-fatal VTE at three months from 1.7 to $0.4 \%$, without any significant increase in the risk of bleeding. The quality of evidence for cancer surgery was stronger. It is recommended that patients undergoing general/abdomino-pelvic surgery for cancer, who stratify as high risk, should receive four weeks (extended duration) thromboprophylaxis, provided that such patients are not high risk for bleeding [4]. Kukreja et al. analysed the benefits of in patients undergoing major urologic surgery for cancer. More than $50 \%$ of VTEs occurred after discharge. They confirmed that four-week EDP is safe and effective in preventing postdischarge VTE in high-risk patients [38]. The American Urological Association recommends that selected very high-risk patients may be considered for post-discharge thromboprophylaxis with either LMWH or warfarin. However, specific recommendations do not exist [25]. The European Association of Urology also recommends four weeks as the optimal duration for thromboprophylaxis [26]. The primary concerns regarding extended duration prophylaxis are the additional cost incurred and the patients' ability to self-administer injectable anticoagulants.

\subsection{Risk factors for VTE}

Multiple risk factors have been identified as playing a role in the development and progression of VTE, and evidence suggests that the number of risk factors present in an individual correlates with an increase in the likelihood of VTE developing. It has previously been shown that the proportion of patients with objective confirmation of DVT increases with the number of individual risk factors present [39].

The elective urology surgical population at $\mathrm{CHBAH}$ had an average of 4.06 risk factors for VTE per person (including surgery as a risk factor). The vast majority of patients (90.7\%) had multiple risk factors. The most frequent risk factors for potential VTE in this population were surgery $(100 \%)$, age $(70.35 \%)$, obesity $(52.87 \%)$, past or present malignancy $(22.12 \%)$ and HIV infection (21.68\%). When accounting for the different weighting of these risk factors on the Caprini RAM, they were also most significant contributors to the total Caprini score. Hence, these are the five most clinically significant risk factors for VTE in this study population. Of these risk factors, only an elevated BMI and HIV are potentially modifiable risk factors.

A study conducted on gynaecological patients in South Africa mirrored the results of this current study, finding that the most frequent risk factors for VTE were surgery, elevated BMI and age [23].

\subsubsection{Major surgery}

Major surgery has been described in the literature as a strong risk factor for VTE, with an odds ratio $>10$ [40]. The population in this study was, by definition, planned for elective surgery. Hence, major surgery, defined as surgery lasting more than $45 \mathrm{~min}$, was the most prevalent risk factor for VTE (67.7\%). A significant proportion of the patients in the sample population were undergoing endoscopic major surgery rather than open surgery. While endoscopic surgery may represent a lower risk for VTE, the difference is not distinguished in the ACCP guideline or the Caprini RAM.

Surgery-related DVT often begins intra-operatively, and may resolve spontaneously by $72 \mathrm{~h}$. The risk of developing post-operative VTE is the highest within the first two weeks, and in particular, the risk for fatal pulmonary embolism is the highest from day three to seven. However, VTE risk remains elevated for at least two to three months [2]. Surgery-related VTE is multifactorial and involves all three components of Virchow's triad. Anaesthetic muscle relaxation, immobility and surgical position may contribute to venous stasis in the lower limb, 
particularly in the region of valves or venous sinuses. Endothelial injury during surgery may result in fibrin deposition in these low-flow areas. Hypoxia, inflammation and changes in haematocrit may result in downregulation of anti-thrombotic proteins, resulting in a hypercoagulable state [41].

\subsubsection{Age}

The risk of VTE rises considerably with age. In particular, persons over the age of 40 are at an increased risk, and this risk appears to double with each decade [40]. The reasons for this are multiple. Older individuals have been shown to have elevated serum levels of prothrombotic proteins. They are also more likely to have other illnesses (such as cancer) and they are likely to have more frequent infections. Relative immobility is also a contributing factor [41].

The median age of the general population in Gauteng Province is around 28 [42]. At CHBAH, both the median and mean age for persons undergoing urology surgery in this study was 52 , suggesting a normally distributed, relatively older surgical population. With $70 \%$ of the population over the age of 40 , the majority of patients studied have a baseline risk for VTE-based purely on their age. Older persons in this study generally scored higher on the Caprini RAM, and thus had a greater number of individual risk factors. Oncology surgery was also more likely to be performed on older individuals. Age, while nonmodifiable, is a significant risk factor for VTE among patients undergoing urology surgery.

\subsubsection{Overweight and obesity}

The 2005 Caprini RAM adds 1 point for all persons with a BMI greater than $25 \mathrm{~kg} / \mathrm{m}^{2}$ and the term "obesity" is used to describe these patients. Subsequent versions of the Caprini RAM consider class III obesity as an independent, more strongly weighted risk factor for VTE. This has not been extensively validated yet $[5,43]$.

For the purpose of this study, the following generally accepted classification has been used: overweight is defined as a BMI between 25.0 and $29.9 \mathrm{~kg} / \mathrm{m}^{2}$, while class I obesity is a BMI of 30.0 to $34.9 \mathrm{~kg} / \mathrm{m}^{2}$, class II obesity a BMI of 35.0 to $39.9 \mathrm{~kg} / \mathrm{m}^{2}$, and class III obesity is a $\mathrm{BMI} \geq 40 \mathrm{~kg} / \mathrm{m}^{2}$ [6]. Being overweight was the most prevalent modifiable risk factor for VTE in the studied population. The majority (57\%) of patients were overweight, and the average BMI was 26.3. Older patients and females were more likely to be obese. In 2008, the mean BMI for the general South African population was estimated to be $26.9 \mathrm{~kg} / \mathrm{m}^{2}$ for males, and $29.5 \mathrm{~kg} / \mathrm{m}^{2}$ for females. These are both higher than the global averages [44].

Overweight individuals may have elevated levels of clotting factors VIII and IX, which may contribute to the increased risk of thrombosis, but the risk of VTE associated with obesity remains, even after adjustment for clotting factor levels [41]. The prothrombotic state seen in these individuals has been attributed to genetic predisposition as well as a pro-inflammatory state as a result of insulin resistance and oxidative stress [45]. The association between VTE and a raised BMI, however appears to be weak, with odds ratios generally below two [40, 45]. A criticism of the use of BMI as a marker for obesity are that other anthropometric measurements such as waist circumference may be better markers for the prediction of cardiovascular disease. BMI may also not correlate with body fat percentage in certain age and race groups [45].

As one of the few common, modifiable risk factors in the population at $\mathrm{CHBAH}$, clinicians should, when possible, encourage patients to implement appropriate lifestyle interventions in order to achieve a healthier BMI prior to elective surgery.

\subsubsection{Malignancy}

Cancer is generally associated with a fourfold increase in the risk of VTE [46]. Tumours shed microparticles which have procoagulant properties. In addition, tumours may cause compression of blood vessels resulting in venous stasis. Oncology patients may also undergo surgery or chemotherapy which increases the risk for VTE, and progression of malignant disease may be associated with relative immobility [41].

Among patients undergoing elective urology surgery at $\mathrm{CHBAH}, 17 \%$ were undergoing surgery for active malignancy and overall, $22 \%$ of patients had a past or present history of cancer. Even when correcting for active malignancy, oncology patients at $\mathrm{CHBAH}$ had higher mean Caprini scores than non-oncology patients.

Clinicians should take particular note of a patients' cancer diagnosis when considering post-operative thromboprophylaxis, as this study shows that these patients almost always require pharmacological thromboprophylaxis and may benefit from extended duration therapy.

\subsubsection{Human immunodeficiency virus infection}

The 2005 Caprini RAM does not specifically list HIV as a risk factor for VTE. However, based on the evidence available as well as personal correspondence with Dr Joseph Caprini, HIV was scored with 1 point under "other risk factors". The 2013 updated Caprini RAM now includes HIV infection as a risk factor weighted to 1 point [47].

The literature suggests that HIV is associated with between a 2 to a 10 times increased risk of VTE. Most of these studies were conducted prior to the widespread roll-out of antiretroviral treatment. HIV positive 
individuals may have deficiencies of protein $\mathrm{C}$ and protein $\mathrm{S}$, as well as elevated levels of tissue factor. This may create a hypercoagulable environment. The virus itself may also cause endothelial damage which predisposes to thrombosis. In addition, the use of protease inhibitors may increase an individuals' risk for VTE. Patients infected with HIV are more likely to acquire opportunistic infections and malignancies, and these may also increase the risk of thrombosis [48]. The risk of VTE in HIV positive individuals appears greater with lower CD4 counts and higher viral loads [48].

Twenty-two per cent of the elective urology population at CHBAH were known to be infected with HIV, which is higher than the $17.6 \%$ of the Gauteng provincial prevalence for adults [47]. The integrity of an HIV positive individuals' immune status could be seen as a potentially modifiable risk factor for VTE, since CD4 counts and viral loads may be influenced by multiple factors, particularly the use of antiretroviral therapy. Given the prevalence of HIV in this sample, it is clear that clinicians should take particular note of a surgical candidates' HIV status, their CD4 count and their viral load as applicable. A consideration would be to delay elective surgery, where possible, until the patients' immune status has been optimised.

\subsubsection{Androgen deprivation therapy}

About $4 \%$ of the population in this study were men on androgen deprivation therapy (c) for prostate cancer. While ADT does not appear as a risk factor on the Caprini RAM, it was considered under "other risk factors" (scoring 1 point). The literature supports this consideration-the risk of VTE in those receiving ADT for prostate cancer is elevated, even when controlling for progression of malignancy. In particular, the risk appears to be higher in patients receiving gonadotropin releasing hormone $(\mathrm{GnRH})$ analogues or in patients following bilateral orchidectomy, rather than patients on anti-androgenic monotherapy. This could be related to the reduction in serum testosterone associated with the aforementioned therapies. Testosterone may have antithrombotic properties [49]. While, the effects of GnRH analogues are generally sustained, ADT could be considered a modifiable risk factor for VTE, particularly in a patient at high risk for VTE whose disease status allowed for a change of drug or cessation of therapy. This study did not document the type of ADT administered, although GnRH analogues are most commonly used at CHBAH.

\subsubsection{Personal or family history of thrombosis}

The Caprini RAM cites a "family history of thrombosis" as the most frequently missed risk factor when assessing
VTE risk [5]. However, very few patients reported a family history or a personal history of thrombosis. This could be due to a lack of awareness regarding the health status of family members in a developing country.

\subsubsection{Obstetric and gynaecological risk factors}

Twenty-one per cent of females in this study had some form of obstetric or gynaecological risk factor for VTE. In this regard, the use of hormonal contraception was the most common risk factor, followed by a history of prior unexplained or recurrent loss of pregnancy. Very few patients were peripartum.

Approximately, 5\% of women of reproductive age have had multiple pregnancy losses. Thrombophilia may be the cause of recurrent loss of pregnancy in up to $40 \%$ of cases, especially in early pregnancy losses. This is often associated with inherited abnormalities of prothrombin or factor $\mathrm{V}$ Leiden. Acquired conditions such as antiphospholipid syndrome may also cause thrombophilia manifesting as a miscarriage or stillbirth [50]. The risk of VTE is elevated in women using oral contraceptives or hormonal replacement therapy. These hormonal therapies may increase plasma fibrinogen and the activity of clotting factors VII and X, while also decreasing antithrombin III activity. In addition, platelet aggregation may increase. The risk of this hypercoagulable state appears to decrease with the duration of therapy, while it is increased by a greater dose of oestrogen within the formulation $[51,52]$. A thorough obstetric and gynaecological history is essential in patients undergoing urological surgery in order to understand whether a patient may have an inherited or acquired thrombophilia.

\subsubsection{Immobility}

Approximately, 5\% of the studied population at $\mathrm{CHBAH}$ had some degree of immobility. Most common were ill patients on bed rest, followed by patients confined to bed for more than three days and patients with longstanding paraparesis.

Post-mortem findings in individuals on prolonged bed rest demonstrate an incidence of VTE of up to $80 \%$. The frequency of VTE appears to increase with the duration of immobility [53]. Other causes of relative immobility which could cause venous stasis and increase an individuals' risk of VTE are lower limb fractures, plaster casts, recent cerebrovascular accidents, recent spinal cord injuries or lower limb surgery. No patients in the sample population demonstrated any of these risk factors. Patients with immobility as a risk factor should be considered for mechanical and/or pharmacological thromboprophylaxis prior to and following urological surgery. Peri-operative ambulation should be encouraged where possible, with the aid of a physiotherapist if necessary. 


\subsubsection{Chronic venous disease}

The term chronic venous disease is used to describe a spectrum of disorders including varicose veins and chronic venous insufficiency (CVI), among others. These disorders are thought to be underdiagnosed, with a prevalence of up to $60 \%$ [54]. Approximately, $10 \%$ of elective urology patients at $\mathrm{CHBAH}$ presented with swollen legs, varicosities or CVI. Swollen legs is a non-specific finding, but it may be a manifestation of CVI and is considered to be a risk for VTE [5]. Dysfunction of lower limb venous drainage may result from incompetence of valves in the deep or superficial veins. Venous obstruction, such as that which occurs in an established DVT, may contribute to this [54]. While it is clear that a DVT may cause chronic venous disease, the role of varicosities in the development of DVT is less clear. There is some evidence to suggest an increased risk for DVT in persons with varicose veins compared with the general population [55]. However, on the whole, varicosities are thought to be a weak risk factor for VTE [40].

\subsection{VTE risk between genders}

In this study, females were more likely to be overweight, while males were more likely to undergo surgery for cancer. Overall, there were no statistically significant differences in the number of risk factors, or the total Caprini score and risk for VTE between males and females at $\mathrm{CHBAH}$. This is in keeping with literature which suggests that overall, VTE risk appears to be equivalent in males and females [56]. While incidence rates appear to be higher in females during their child bearing years, the incidence after the age of 45 is higher in men [57].

\section{Conclusions}

Venous thromboembolism is an important preventable cause of morbidity and mortality in urology patients. The disease may be difficult to diagnose, and clinicians may underestimate the risk for developing VTE. Risk assessment models, such as the Caprini score, are objective and practical tools to guide the application of thromboprophylaxis. This is the first African study evaluating the risk for post-operative VTE in an exclusively urological population. The application of the Caprini RAM in the elective urological surgery population at Chris Hani Baragwanath Academic Hospital yields similar results to studies performed elsewhere on similar surgical populations. Age, malignancy, major surgery, HIV infection and a raised BMI are the most important risk factors in this population. Elective urology patients are at moderate to high risk of post-operative VTE, and some form of prophylaxis is required for almost all patients. Patients undergoing urology surgery for cancer are, in particular, at high risk, and should be considered for extended duration pharmacological thromboprophylaxis following discharge.

Limitations of this study include the relatively small sample size and non-consecutive sampling. It is also unclear whether the Caprini risk assessment model accurately predicts VTE in a population with a high proportion of endoscopic procedures. Further research is required to evaluate whether the incidence of VTE correlates with the risk assessment in this population. Clinician compliance with the use of RAMs as well as the corresponding recommendations for prophylaxis need to be evaluated. A validated risk assessment model which accounts for procedure-specific risks in urology may be useful.

\section{Abbreviations}

VTE: Venous thromboembolism; DVT: Deep vein thrombosis; PE: Pulmonary embolism; RAM: Risk assessment model; ACCP: American College of Chest Physicians; CHBAH: Chris Hani Baragwanath Academic Hospital; AUA: American Urological Association; EAU: European Association of Urology; ROTBUS: Risk of Thrombosis and Bleeding in Urological Surgery; TURP: Transurethral resection of the prostate; LMWH: Low molecular weight heparin; ADT: Androgen deprivation therapy; CVI: Chronic venous insufficiency.

\section{Acknowledgements \\ Sincere thanks to Christopher Manyamba for his assistance with statistical analysis.}

\section{Authors' contributions}

All authors approved the final version of the manuscript for submission. WPRP conceived the study, obtained ethical approval, drafted, reviewed for critical content, and edited the manuscript. MN supervised, reviewed for critical content and edited the manuscript. MH supervised, reviewed for critical content. All authors read and approved the final manuscript.

Funding

No external funding was required.

Availability of data and materials

The datasets used and/or analysed during the current study are available from the corresponding author on reasonable request.

\section{Declarations}

\section{Ethics approval and consent to participate}

Information was collected by interviewing/examining patients and reviewing available clinical records. Written informed consent from study participants was obtained. This study was approved by the University of Witwatersrand Human Research Ethics Committee (protocol no: M191175). Permission for the study was obtained from the relevant clinical head of department and the $\mathrm{CHBAH}$ chief executive officer.

\section{Consent for publication}

Not applicable.

\section{Competing interests}

None.

\section{Author details}

${ }^{1}$ Division of Urology, Department of Surgery, School of Clinical Medicine, Faculty of Health Sciences, University of the Witwatersrand, Johannesburg, South Africa. ${ }^{2}$ Department of Surgery, School of Clinical Medicine, Faculty of Health Sciences, University of the Witwatersrand, Johannesburg, South Africa. 
Received: 18 May 2021 Accepted: 6 September 2021

Published online: 20 September 2021

\section{References}

1. Kumar DR, Hanlin E, Glurich I, Mazza JJ, Yale SH (2010) Virchow's contribution to the understanding of thrombosis and cellular biology. Clin Med Res 8(3-4):168-172

2. Kearon C (2003) Natural history of venous thromboembolism. Circulation 107(90231):221 - 30

3. Moheimani F, Jackson DE (2011) Venous thromboembolism: classification, risk factors, diagnosis, and management. ISRN Hematol 2011:1-7

4. Gould MK, Garcia DA, Wren SM, Karanicolas PJ, Arcelus JI, Heit JA et al (2012) Prevention of VTE in nonorthopedic surgical patients. Chest 141(2):e227S-e277S

5. Caprini JA (2005) Thrombosis risk assessment as a guide to quality patient care. Dis Mon 51(2-3):70-78

6. Becker $\mathrm{KL}$ (2001) Principles and practice of endocrinology and metabolism. Lippincott Williams \& Wilkins, p 2500

7. Beckman MG, Hooper WC, Critchley SE, Ortel TL (2010) Venous thromboembolism. Am J Prev Med 38(4):S495-501

8. Goldhaber SZ (2012) Venous thromboembolism: epidemiology and magnitude of the problem. Best Pract Res Clin Haematol 25(3):235-242

9. Cohen A, Agnelli G, Anderson F, Arcelus J, Bergqvist D, Brecht J et al (2007) Venous thromboembolism (VTE) in Europe: the number of VTE events and associated morbidity and mortality. Thromb Haemost 98(10):756-764

10. Blann AD, Lip GYH (2006) Venous thromboembolism. BMJ 332(7535):215-219

11. Jacobson BF, Louw S, Büller H, Mer M, De Jong PR, Rowji P et al (2013) Venous thromboembolism: prophylactic and therapeutic practice guideline. S Afr Med J 103(4):260

12. Tagalakis V, Patenaude V, Kahn SR, Suissa S (2013) Incidence of and mortality from venous thromboembolism in a real-world population: the Q-VTE study cohort. Am J Med 126(9):832.e13-832.e21

13. Danwang C, Temgoua MN, Agbor VN, Tankeu AT, Noubiap JJ (2017) Epidemiology of venous thromboembolism in Africa: a systematic review. J Thromb Haemost 15(9):1770-1781

14. Bahl V, Hu HM, Henke PK, Wakefield TW, Campbell DA, Caprini JA (2010) A validation study of a retrospective venous thromboembolism risk scoring method. Ann Surg 251(2):344-350

15. Pannucci CJ, Bailey SH, Dreszer G, Fisher Wachtman C, Zumsteg JW, Jaber RM et al (2011) Validation of the Caprini risk assessment model in plastic and reconstructive surgery patients. J Am Coll Surg 212(1):105-112

16. Shuman AG, Hu HM, Pannucci CJ, Jackson CR, Bradford CR, BahI V (2012) Stratifying the risk of venous thromboembolism in otolaryngology. Otolaryngol Head Neck Surg 146(5):719-724

17. Obi AT, Pannucci CJ, Nackashi A, Abdullah N, Alvarez R, Bahl V et al (2015) Validation of the Caprini venous thromboembolism risk assessment model in critically III surgical patients. JAMA Surg 150(10):941

18. Macht R, Gardner I, Talutis S, Rosenkranz P, Doherty G, McAneny D (2017) Evaluation of a standardized risk-based venous thromboembolism prophylaxis protocol in the setting of thyroid and parathyroid surgery. J Am Coll Surg 224(6):1029-1035

19. Frankel JK, Belanger M, Tortora J, McLaughlin T, Staff I, Wagner J (2020) MP47-17 Caprini score predicts venous thromboembolic events in patients undergoing robotic assisted prostatectomy. J Urol. https://doi. org/10.1016/j.juro.2017.02.1477

20. McAlpine K, Breau RH, Mallick R, Cnossen S, Cagiannos I, Morash C et al (2017) Current guidelines do not sufficiently discriminate venous thromboembolism risk in urology. Urol Oncol Semin Original Investig 35(7):457. e1-457.e8

21. Pannucci CJ, Swistun L, MacDonald JK, Henke PK, Brooke BS (2017) Individualized venous thromboembolism risk stratification using the 2005 Caprini score to identify the benefits and harms of chemoprophylaxis in surgical patients: a meta-analysis. Ann Surg 265(6):1094-1103

22. Barber EL, Clarke-Pearson DL (2016) The limited utility of currently available venous thromboembolism risk assessment tools in gynecological oncology patients. Am J Obstet Gynecol 215(4):445.e1-445.e9
23. Snyman LC, Potgieter J (2014) Venous thromboembolism: risk profile and management of prophylaxis in gynaecological surgery patients. S Afr J OG 20(3):76

24. Bilgi K, Muthusamy A, Subair M, Srinivasan S, Kumar A, Ravi R et al (2016) Assessing the risk for development of Venous thromboembolism (VTE) in surgical patients using adapted Caprini scoring system. Int J Surg 30:68-73

25. Forrest JB, Clemens JQ, Finamore P, Leveillee R, Lippert M, Pisters $L$ et al (2009) AUA best practice statement for the prevention of deep vein thrombosis in patients undergoing urologic surgery. J Urol 181(3):1170-1177

26. Professionals S-O. EAU guidelines: thromboprophylaxis [internet]. Uroweb. [cited 2020 Sep 2]. Available from: https://uroweb.org/guide line/thromboprophylaxis/

27. Violette PD, Cartwright R, Briel M, Tikkinen KAO, Guyatt GH (2016) Guideline of guidelines: thromboprophylaxis for urological surgery. BJU Int 118(3):351-358

28. Geerts WH, Heit JA, Clagett GP, Pineo GF, Colwell CW, Anderson FA et al (2001) Prevention of venous thromboembolism. Chest 119(1):132S-175S

29. Kohro S, Yamakage M, Takahashi T, Kondo M, Ota K, Namiki A (2002) Intermittent pneumatic compression prevents venous stasis in the lower extremities in the lithotomy position. Can J Anesth 49(2):144-147

30. Scarpa RM, Carrieri G, Gussoni G, Tubaro A, Conti G, Pagliarulo V et al (2007) Clinically overt venous thromboembolism after urologic cancer surgery: results from the @RISTOS Study. Eur Urol 51(1):130-5; discussion 136

31. Clément C, Rossi P, Aissi K, Barthelemy P, Guibert N, Auquier P et al (2011) Incidence, risk profile and morphological pattern of lower extremity venous thromboembolism after urological cancer surgery. J Uro 186(6):2293-2297

32. Alberts BD, Woldu SL, Weinberg AC, Danzig MR, Korets R, Badani KK (2014) Venous thromboembolism after major urologic oncology surgery: a focus on the incidence and timing of thromboembolic events after 27,455 operations. Urology 84(4):799-807

33. Tikkinen KA, Agarwal A, Craigie S, Cartwright R, Gould MK, Haukka J et al (2014) Systematic reviews of observational studies of risk of thrombosis and bleeding in urological surgery (ROTBUS): introduction and methodology. Syst Rev 3(1):150

34. Tikkinen $\mathrm{KAO}$, Craigie $S$, Agarwal A, Violette PD, Novara G, Cartwright R et al (2018) Procedure-specific risks of thrombosis and bleeding in urological cancer surgery: systematic review and meta-analysis. Eur Urol 73(2):242-251

35. Tikkinen KAO, Craigie S, Agarwal A, Siemieniuk RAC, Cartwright R, Violette PD et al (2018) Procedure-specific risks of thrombosis and bleeding in urological non-cancer surgery: systematic review and meta-analysis. Eur Urol 73(2):236-241

36. Saluja M, Gilling P (2017) Venous thromboembolism prophylaxis in urology: a review. Int J Urol 24(8):589-593

37. White R, Zhou H, Romano P (2003) Incidence of symptomatic venous thromboembolism after different elective or urgent surgical procedures. Thromb Haemost 90(09):446-455

38. Kukreja JEB, Levey HR, Scosyrev E, Kiernan M, Berrondo C, McNamee C et al (2015) Effectiveness and safety of extended-duration prophylaxis for venous thromboembolism in major urologic oncology surgery. Urol Oncol Semin Original Investig 33(9):387.e7-387.e16

39. Wheeler HB (1982) Suspected deep vein thrombosis: management by impedance plethysmography. Arch Surg 117(9):1206

40. Anderson FA (2003) Risk factors for venous thromboembolism. Circulation 107(90231):91 - 16

41. Esmon CT (2009) Basic mechanisms and pathogenesis of venous thrombosis. Blood Rev 23(5):225-229

42. Statistics South Africa. Census 2011: population dynamics in South Africa. 2015

43. Cronin M, Dengler N, Krauss ES, Segal A, Wei N, Daly M et al (2019) Completion of the updated Caprini risk assessment model (2013 version). Clin Appl Thromb Hemost 25:107602961983805

44. Finucane MM, Stevens GA, Cowan MJ, Danaei G, Lin JK, Paciorek CJ et al (2011) National, regional, and global trends in body-mass index since 1980: systematic analysis of health examination surveys and epidemiological studies with 960 country-years and 9.1 million participants. Lancet 377(9765):557-567 
45. Yang G, Staercke CD, Hooper WC (2012) The effects of obesity on venous thromboembolism: a review. OJPM 02(04):499-509

46. Lee AYY (2003) Venous thromboembolism and cancer: risks and outcomes. Circulation 107(90231):17I - 21

47. South African National HIV Prevalence, Incidence, Behaviour and Communication Survey, 2017-The Human Sciences Research Council (HSRC) [Internet]. [cited 2020 Aug 31]. Available from: https://www.hsrcpress.ac. za/books/south-african-national-hiv-prevalence-incidence-behaviourand-communication-survey-2017

48. Bibas M, Biava G, Antinori A (2011) HIV-associated venous thromboembolism. Mediterr J Hematol Infect Dis 3(1):2011030

49. O'Farrell S, Sandström K, Garmo H, Stattin P, Holmberg L, Adolfsson J et al (2016) Risk of thromboembolic disease in men with prostate cancer undergoing androgen deprivation therapy. BJU Int 118(3):391-398

50. D'Uva M (2010) Recurrent pregnancy loss and thrombophilia. J Clin Med Res [Internet]. [cited 2020 Sep 1]. Available from: http://www.jocmr.org/ index.php/JOCMR/article/view/260

51. Bonnar J (1987) Coagulation effects of oral contraception. Am J Obstet Gynecol 157(4):1042-1048
52. Lidegaard O, Lokkegaard E, Svendsen AL, Agger C (2009) Hormonal contraception and risk of venous thromboembolism: national follow-up study. BMJ 339(2):2890-2890

53. Gibbs NM (1957) Venous thrombosis of the lower limbs with particular reference to bed-rest. Br J Surg 45(191):209-236

54. Eberhardt RT, Raffetto JD (2014) Chronic venous insufficiency. Circulation 130(4):333-346

55. Müller-Bühl L, Engeser AN, Szecsenyi L (2012) Varicose veins are a risk factor for deep venous thrombosis in general practice patients. Vasa 41(5):360-365

56. White RH (2003) The epidemiology of venous thromboembolism. Circulation 107(90231):4l - 8

57. Heit JA, Spencer FA, White RH (2016) The epidemiology of venous thromboembolism. J Thromb Thrombol 41(1):3-14

\section{Publisher's Note}

Springer Nature remains neutral with regard to jurisdictional claims in published maps and institutional affiliations.

\section{Submit your manuscript to a SpringerOpen ${ }^{\circ}$ journal and benefit from:}

- Convenient online submission

- Rigorous peer review

- Open access: articles freely available online

- High visibility within the field

- Retaining the copyright to your article

Submit your next manuscript at $\boldsymbol{\nabla}$ springeropen.com 